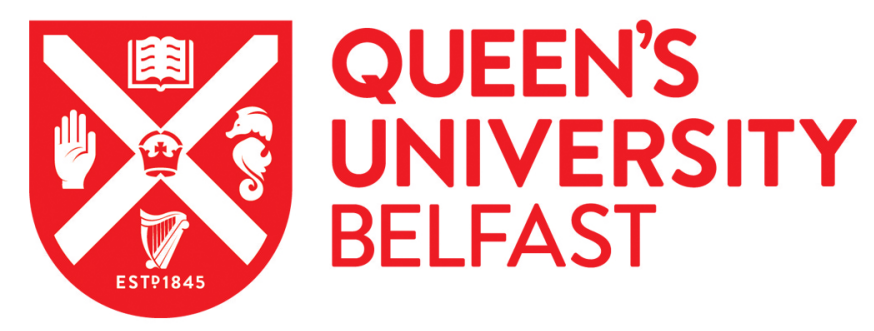

\title{
SN2017jgh - A high-cadence complete shock cooling lightcurve of a SN IIb with the Kepler telescope
}

Armstrong, P., Tucker, B. E., Rest, A., Ridden-Harper, R., Zenati, Y., Piro, A. L., Hinton, S., Lidman, C., Margheim, S., Narayan, G., Shaya, E., Garnavich, P., Kasen, D., Villar, V., Zenteno, A., Arcavi, I., Drout, M., Foley, R. J., Wheeler, J., ... Zsidi, G. (2021). SN2017jgh - A high-cadence complete shock cooling lightcurve of a SN Ilb with the Kepler telescope. Monthly Notices of the Royal Astronomical Society, 507(3), 3125-3138. https://doi.org/10.1093/mnras/stab2138, https://doi.org/10.1093/mnras/stab2138

Published in:

Monthly Notices of the Royal Astronomical Society

Document Version:

Publisher's PDF, also known as Version of record

Queen's University Belfast - Research Portal:

Link to publication record in Queen's University Belfast Research Portal

Publisher rights

Copyright 2021 The Author(s) Published by Oxford University Press on behalf of Royal Astronomical Society.

This work is made available online in accordance with the publisher's policies. Please refer to any applicable terms of use of the publisher.

\section{General rights}

Copyright for the publications made accessible via the Queen's University Belfast Research Portal is retained by the author(s) and / or other copyright owners and it is a condition of accessing these publications that users recognise and abide by the legal requirements associated with these rights.

Take down policy

The Research Portal is Queen's institutional repository that provides access to Queen's research output. Every effort has been made to ensure that content in the Research Portal does not infringe any person's rights, or applicable UK laws. If you discover content in the Research Portal that you believe breaches copyright or violates any law, please contact openaccess@qub.ac.uk. 


\title{
SN2017jgh: a high-cadence complete shock cooling light curve of a SN IIb with the Kepler telescope
}

\author{
P. Armstrong ${ }^{(0,}{ }^{1 \star}$ B. E. Tucker, ${ }^{1,2,3 \star}$ A. Rest, ${ }^{4,5 \star}$ R. Ridden-Harper, ${ }^{4,5}$ Y. Zenati, ${ }^{5}$ A. L. Piro, ${ }^{6}$ \\ S. Hinton ${ }^{\circledR},{ }^{7}$ C. Lidman ${ }^{\circledR},{ }^{1,8}$ S. Margheim, ${ }^{9}$ G. Narayan, ${ }^{10,11}$ E. Shaya, ${ }^{12}$ P. Garnavich ${ }^{\circledR}, 13$ \\ D. Kasen, ${ }^{14,15,16}$ V. Villar, ${ }^{17}$ A. Zenteno ${ }^{\circledR},{ }^{18}$ I. Arcavi, ${ }^{19,20}$ M. Drout, ${ }^{6,21}$ R. J. Foley, ${ }^{22}$ J. Wheeler ${ }^{(0)},{ }^{23}$ \\ J. Anais,${ }^{24}$ A. Campillay, ${ }^{25}$ D. Coulter, ${ }^{22}$ G. Dimitriadis, ${ }^{22}$ D. Jones, ${ }^{22}$ C. D. Kilpatrick, ${ }^{26,27}$ \\ N. Muñoz-Elgueta, ${ }^{28}$ C. Rojas-Bravo, ${ }^{22}$ J. Vargas-González, ${ }^{29}$ J. Bulger, ${ }^{30}$ K. Chambers, ${ }^{30}$ M. Huber ${ }^{\circledR}, 30$ \\ T. Lowe, ${ }^{30}$ E. Magnier, ${ }^{30}$ B. J. Shappee, ${ }^{30}$ S. Smartt, ${ }^{31}$ K. W. Smith ${ }^{\circledR},{ }^{31}$ T. Barclay, ${ }^{32,33}$ G. Barentsen, ${ }^{34}$ \\ J. Dotson, ${ }^{35}$ M. Gully-Santiago, ${ }^{23}$ C. Hedges, ${ }^{34,35}$ S. Howell, ${ }^{35}$ A. Cody, ${ }^{35}$ K. Auchettl, ${ }^{3,22,36}$ A. Bódi, ${ }^{37,38}$ \\ Zs. Bognár, ${ }^{37,38}$ J. Brimacombe, ${ }^{39}$ P. Brown, ${ }^{40}$ B. Cseh, ${ }^{37}$ L. Galbany ${ }^{\circledR},{ }^{41}$ D. Hiramatsu, ${ }^{42,43}$ \\ T. W.-S. Holoien, ${ }^{6}$ D. A. Howell, ${ }^{42,43}$ S. W. Jha ${ }^{\circledR}{ }^{44}$ R. Könyves-Tóth, ${ }^{37,45}$ L. Kriskovics, ${ }^{37}$ \\ C. McCully, ${ }^{42,43}$ P. Milne, ${ }^{46}$ J. Muñoz, ${ }^{47,48}$ Y. Pan, ${ }^{49}$ A. Pál, ${ }^{37}$ H. Sai, ${ }^{50}$ K. Sárneczky, ${ }^{37}$ N. Smith ${ }^{\circledR}, 46$ \\ Á. Sódor, ${ }^{37,38}$ R. Szabó, ${ }^{37,38,51}$ R. Szakáts, ${ }^{37}$ S. Valenti, ${ }^{52}$ J. Vinkó, ${ }^{23,37,45,51}$ X. Wang, ${ }^{50,53}$ K. Zhang ${ }^{\circledR 54}$ \\ and G. Zsidi ${ }^{37,51,55}$
}

Affiliations are listed at the end of the paper

Accepted 2021 July 15. Received 2021 June 29; in original form 2021 March 31

\begin{abstract}
SN 2017jgh is a type IIb supernova discovered by Pan-STARRS during the C16/C17 campaigns of the Kepler/K2 mission. Here, we present the Kepler/K2 and ground based observations of SN 2017jgh, which captured the shock cooling of the progenitor shock breakout with an unprecedented cadence. This event presents a unique opportunity to investigate the progenitors of stripped envelope supernovae. By fitting analytical models to the SN 2017jgh light curve, we find that the progenitor of SN 2017jgh was likely a yellow supergiant with an envelope radius of $\sim 50-290 \mathrm{R}_{\odot}$, and an envelope mass of $\sim 0-1.7 \mathrm{M}_{\odot}$. SN 2017jgh likely had a shock velocity of $\sim 7500-10300 \mathrm{~km} \mathrm{~s}^{-1}$. Additionally, we use the light curve of SN $2017 \mathrm{jgh}$ to investigate how early observations of the rise contribute to constraints on progenitor models. Fitting just the ground based observations, we find an envelope radius of $\sim 50-330 \mathrm{R}_{\odot}$, an envelope mass of $\sim 0.3-1.7 \mathrm{M}_{\odot}$ and a shock velocity of $\sim 9000-15000 \mathrm{~km} \mathrm{~s}-1$. Without the rise, the explosion time cannot be well constrained that leads to a systematic offset in the velocity parameter and larger uncertainties in the mass and radius. Therefore, it is likely that progenitor property estimates through these models may have larger systematic uncertainties than previously calculated.
\end{abstract}

Key words: shock waves - supernovae: general - supernovae: individual - transients: supernovae.

\section{INTRODUCTION}

Massive stars end their lives as a core-collapse supernovae (CCSNe), leaving behind a black hole or neutron star and a supernova remnant. CCSNe come in a variety of observed sub-types depending on the properties of the progenitor star prior to explosion, the explosion mechanism itself, and its circumstellar environment. Type II supernovae display strong hydrogen lines in their early spectra, with this population consisting of Type II-P and II-L SNe (plateauing and linear light-curve decay, respectively), Type IIn SNe (narrow line spectra), and Type IIb SNe (He in spectrum with disappearing $\mathrm{H}$;

^E-mail: patrick.armstrong@anu.edu.au (PA); brad.tucker@anu.edu.au (BET); arest@stsci.edu (AR)
Filippenko 1997). Type I supernovae lack strong H lines, such as Type Ib SNe (He in spectrum), and Type Ic SNe (no He in spectrum; Janka 2012; Gal-Yam 2017). SNe IIb along with Ib and Ic are known as stripped envelope supernova as they lose their hydrogen over time.

Weeks after the explosion of Type IIb Sne, hydrogen lines begin to disappear and helium lines begin to dominate with the spectrum more closely resembling a type Ib supernova (Filippenko 1997), suggesting a progenitor which is mostly stripped of its hydrogen envelope. The mechanism which strips the hydrogen is still unknown, with possibilities including stellar winds, stellar rotation, binary interaction, and nuclear burning instabilities (e.g. Podsiadlowski 1992; Woosley, Langer \& Weaver 1993; Bersten et al. 2012; Liu et al. 2016; Yoon, Dessart \& Clocchiatti 2017; Bersten et al. 2018; Naiman et al. 2019; Sravan, Marchant \& Kalogera 2019). 
Type IIb SNe are of particular interest as many IIb SNe progenitors have been identified. Several have evidence of binary interaction (e.g. SN 1993J; Maund et al. 2004, SN 2011dh; Bersten et al. 2012). A well-studied example of a type IIb supernova is SN 1993J (Nomoto et al. 1993; Richmond et al. 1994). The progenitor of this supernova was identified from direct imaging as a late $\mathrm{G}$ or early $\mathrm{K}$ supergiant with an effective temperature between 4000 and $4500 \mathrm{~K}$ and an initial mass prior to explosion of $17 \mathrm{M}_{\odot}$. There is evidence that the progenitor of SN 1993J had a binary companion that may be responsible for stripping the progenitor of its hydrogen (Maund et al. 2004).

Stripped supernovae can show two prominent peaks in their optical light curve. The first peak is a burst of emission after the explosion known as the shock cooling light curve (SCL; Arcavi 2017; Gal-Yam 2017; Sravan et al. 2020), and the second peak is nuclear powered emission fuelled by the decay of ${ }^{56} \mathrm{Ni}$.

The SCL provides an opportunity to probe physical properties of the progenitor. This emission is produced when photons trapped behind optically dense material within the progenitor finally escape (Waxman \& Katz 2017). The associated optical emission lasts on the order of days, and the shape of this curve depends on both the behaviour of the shock wave and the physical properties of the progenitor star (Nakar \& Sari 2010; Waxman \& Katz 2017 both provide a more complete review of shock breakout physics). As such, investigating the SCL provides a unique opportunity to probe both the properties of the shock wave and the progenitor star.

There have been many supernovae observed with a SCL - SN 1993J (Richmond et al. 1994), SN 2011dh (Arcavi et al. 2011), SN 2011fu (Kumar et al. 2013), SN 2011hs (Bufano et al. 2014), SN 2013df (Morales-Garoffolo et al. 2014), and SN 2016gkg (Arcavi et al. 2017; Kilpatrick et al. 2017). Hydrodynamical modelling of SN 2011dh suggests that a star with a compact core and low-mass envelope is most likely responsible for the observed SCL (Bersten et al. 2012). This was later confirmed by Nakar \& Piro (2014) who employed semi-analytical models to show that a progenitor with an extended envelope was required. The Nakar \& Piro (2014) model includes two relationships: one between the bolometric luminosity at the peak of the SCL and the radius of the extended envelope, and the other between the time of the peak of the SCL and the mass concentrated at the envelope's radius. Piro (2015, hereafter P15) produced an analytical expression for the complete SCL. P15 enforced conditions necessary to produce a SCL that require that the material is massive enough for the shock to propagate and extended enough for the peak to be bright in the optical band. No explicit assumptions are made as to the density profile of the supernova progenitor. An alternative to the P15 model, Rabinak \& Waxman (2011), explicitly assume a polytropic density profile to provide a more physically realistic model of the progenitor. This was expanded upon by Sapir \& Waxman (2017) (hereafter SW17) by scaling their model to better agree with hydrodynamical simulations, allowing the model to extend to later times. Piro, Haynie \& Yao (2020, hereafter P20), like the P15 model, makes no assumptions about the density profile of the progenitor star, instead the P20 model makes use of a two-component velocity profile to attain better fits to the SCL than P15. All attempts at modelling the SCL thus far rely on the assumption of hydrostatic equilibrium within the progenitor (Chevalier 1993; Nakar \& Sari 2010).

Capturing the shock cooling light curve of a supernova is difficult owing to the short lifetime of these events. Most examples of SCLs have only included the decline of the SCL, sometimes managing to capture the peak as well. The rise of the SCL was yet to be captured at high cadence, which presents a problem for the development and improvement of SCL models and, thus, our ability to probe the progenitor of these supernovae. As mentioned in P15, the shape of the rise is highly dependent on the density structure of the progenitor which makes the rise difficult to accurately model. Without data of the SCL rise, one cannot test how effective models are at this critical early stage (Piro 2015; Sapir \& Waxman 2017; Piro et al. 2020).

In order to effectively capture the complete SCL, continuous, high cadence observations are needed. A cadence of $<1 \mathrm{~d}$ allows one to capture the earliest emission from the supernova, and high cadence observations are required in order to observe the rapidly evolving SCL. Telescopes like the Kepler Space Telescope (Kepler/K2; Howell et al. 2014) and the Transiting Exoplanet Survey Satellite (TESS; Ricker et al. 2014) allow for this type of observing strategy. As such, we can expect more shock cooling light curves with higher cadence data to be observed, presenting an exciting opportunity to gain a much better understanding of the progenitors of type IIb supernovae (Fausnaugh et al. 2021; Vallely et al. 2021). In order to effectively use this new data, we need to determine which class of analytical SCL models fits the data the best and how much of the SCL must be observed to constrain progenitor models.

In this paper, we present SN 2017jgh, a type IIb supernova that was observed by Kepler/K2 in Campaign 16. Kepler/K2 was able to capture the full light curve of SN 2017jgh, including the complete evolution of the SCL. This is the first time a high cadence, complete shock cooling light curve has been observed. This represents a unique opportunity to evaluate the effectiveness of the analytical models and to investigate how important the rise is for accurate modelling of progenitor properties.

\section{OBSERVATIONS}

SN 2017jgh ${ }^{1}$ was discovered by Pan-STARRS1 (PS1; Chambers et al. 2016) on 2017 Dec 23 at 11:09:36 (MJD 58110.965) with $g$ $=20.21 \mathrm{mag}$ (Chambers et al. 2017). SN 2017jgh occurred at $(\alpha, \delta)$ $=\left(09^{\mathrm{h}} 02^{\mathrm{m}} 56^{\mathrm{s}} .736,+12^{\circ} 03^{\prime} 04^{\prime \prime} 27\right)$, at a separation of 0.157 arcsec from the centre of its host galaxy, 2MASX J09025612+1202596 with redshift 0.079 . We estimated the Milky Way reddening towards SN 2017jgh as $E(B-V)=0.02$ mag (Schlegel, Finkbeiner \& Davis 1998). We do not take into account host galaxy extinction. This extinction is likely to redden the light curve; however, the shape of the SCL is more important than the colour when fitting so we believe the effects of this extinction to be negligible. We measure the radioactive maximum in the Kepler/K2 band to be $t_{\max }=58127 \pm 1$ MJD. Throughout the paper, epochs are presented relative to this maximum, as $t-t_{\max }$.

\subsection{Ground-based photometry}

Ground-based photometry was obtained from both PS1 (Chambers et al. 2016; Dotson et al. 2018) and the Coulter et al. (2017).

PS1 is a $1.8 \mathrm{~m}$ telescope located at Haleakala on Maui, Hawaii. The telescope contains a 1.4 gigapixel camera, GPC1, mounted at the Cassegrain $f / 4.4$ focus. GPC1 has sixty orthogonal transfer array devices, each with a $4846 \times 4846$ pixel detector area. Each pixels measures $10 \mu \mathrm{m}$ in size, giving a focal plane of $418.88 \mathrm{~mm}$ in diameter $\left(3.3^{\circ}\right)$. This gives a field of view 7.06 square degrees. PS1's filter system ( rizy) is similar to SDSS (Abazajian et al. 2009), with the addition of a composite gri 'wide' filter $w$ (Tonry et al. 2012; Chambers et al. 2016).

\footnotetext{
${ }^{1}$ EPIC: 211427218.
} 
Reduction of PS1 images (described in detail in Magnier et al. 2020) is performed by the PS1 Image Processing Pipeline (IPP; Ryan 2006; Magnier et al. 2008) that includes standard reductions, astrometric solution, stacking of nightly images, source detection, and photometry. The stacks are then sent to the Harvard FAS Cannon Research Computing cluster, where the PHOTPIPE pipeline (Rest et al. 2005) performs difference imaging and transient identification.

SSS's optical photometry of SN 2017jgh was obtained in gri with the Swope 1-meter telescope at Las Campanas, Chile, from 2017 December 17 to 2018 February 7. Following the description in Kilpatrick et al. (2018), all image processing and optical photometry on the Swope data was performed using PHOTPIPE (Rest et al. 2005). The photometry were calibrated using standard sources from the PanSTARRS DR1 catalogue (Flewelling et al. 2020) in the same field as SN 2017jgh and transformed following the Supercal method (Scolnic et al. 2015) into the Swope natural system (Krisciunas et al. 2017). Deep gri template images of the SN 2017jgh field were obtained on 2019 January 8 and January 14. Final image subtraction was performed using HOTPANTS (Becker 2015). Forced photometry was performed on the subtracted images.

\subsection{Kepler/K2 photometry}

The K2 mission consists of a series of campaigns which observe different fields within the ecliptic plane. Kepler/K2 uses a $0.95 \mathrm{~m}$ aperture Schmidt telescope, orbiting the Earth in a heliocentric orbit. The telescope has one broad filter spanning 4000-9000 A, with peak transmission roughly coinciding with the Pan-STARRS $r$ band (Bryson et al. 2010).

The primary advantage of Kepler/K2 is the 30 min cadence. This rapid cadence, combined with the roughly $80 \mathrm{~d}$ campaigns, allows for early and detailed light curves of transient events. Throughout campaign 16, the Kepler spacecraft had been running with the loss of two reaction wheels, so was operating on two remaining wheels and thrusters. This introduced a number of systematic effects into the light curve. These included reaction wheel jitter, which introduces additional short-term noise, and solar pressure induced drifting, which introduced long-term systematics with a characteristic 'sawtooth' pattern.

As described in Shaya, Olling \& Mushotzky (2015), the datareduction pipeline begins by correcting the CCD images for bias level, dark current, smear, non-linear gain, undershooting pixels, and flat fields (Quintana et al. 2010). After initial calibration, the Presearch Data Conditioning (PDC; Stumpe et al. 2012) applies corrections for both instrumental and spacecraft anomalies, and removes contamination from nearby stars. During this process, a set of 14 cotrending basis vectors (CBVs) are generated by singular value decomposition to represent correlated instrumental artifacts such as flexing of the telescope structure, thermal transients, and drifting which occurs due to solar pressure. The PDC light curve then has a superposition of these CBVs removed such that the root-mean-squared (rms) deviations are minimized. However, for $\mathrm{SNe}$, this process would remove most of the physical variations in the light curve. We therefore carefully create a set of CBVs using only quiet galaxies that are on the same CCD channel as the $\mathrm{SN}$ itself. Then we solve for the coefficients of a superposition of $\mathrm{CBV}$ s that minimize the rms deviations before the onset of the $\mathrm{SN}$ event.

In order to calculate the Kepler/K2 zero-point, we follow the Kepler Instrument Handbook (Van Cleve \& Caldwell 2016) that gives the following equation for the Kepler/K2 magnitude $\left(K_{p}\right)$ in terms of the $(g-r)$ colour (where $g$ and $r$ are the SDSS filters):

$K_{p}=\left\{\begin{array}{ll}r+0.2(g-r) & \text { if }(g-r) \leq 0.8 \mathrm{mag} \\ r+0.1(g-r) & \text { if }(g-r)>0.8 \mathrm{mag}\end{array}\right.$.

The $(g-r)$ colour is calculated from the ground-based photometry. Once the pseudo-Kepler/K2 magnitude is calculated, it is compared to the raw Kepler/K2 flux in order to calculate the Kepler/K2 zeropoint. We calculate the Kepler/K2 zero-point to be $25.3 \pm 0.1$. We validate this zero-point by calculating synthetic Kepler photometry, using the Kepler bandpass listed on SVO filter profile service (Rodrigo, Solano \& Bayo 2012; Rodrigo \& Solano 2020), normalized to AB magnitudes. Since the GMO-S spectrum discussed in Section 2.3 did not cover the full wavelength range, we use the SN $2016 \mathrm{gkg}$ spectra taken $2 \mathrm{~d}$ before radioactive maximum by Jha, Van Wyk \& Vaisanen (2016), normalized to the ground-based photometry for SN 2017jgh at the same phase. Comparing to the Kepler counts, we find a zero-point of $23.33 \pm 0.15$, which is within 10 per cent of the zero-point derived from ground-based photometry. Since there are unknown differences between the SN 2017jgh and SN $2016 \mathrm{gkg}$ spectra, we use the ground-based photometric zero-point of $25.3 \pm 0.1$.

The combined Kepler/K2 and ground-based light curve is presented in Fig. 1. The double-peaked profile is evident in the Kepler/K2 photometry and is mirrored in the ground-based data, although the ground-based data is significantly sparser and does not cover the rise. A comparison between SN 2017jgh, SN 1993J (Richmond et al. 1994), and SN 2016gkg (Arcavi et al. 2017) is presented in Fig. 2, and the evolving colours of SN 2017jgh, SN 2016gkg, and SN 1993J are shown in Fig. 3. ${ }^{2}$

The light curve of SN 2017jgh appears most similar to SN 1993J, which has a very similar SCL decline. The SCL of SN 2016gkg has a much sharper decline, which may indicate it had less extended material. Overall, this suggests that the progenitor of SN 2017jgh was closer to the yellow supergiant progenitor of SN 1993J than the blue supergiant progenitor of SN 2016gkg. Additionally, SN 1993J's colour evolution closely matches the $g-r$ colour evolution of SN 2017jgh, which again suggests that the progenitor of SN 1993J and SN 2017jgh were very similar.

An excerpt of the light-curve data is shown in Table 1, with the full data set available online.

\subsection{Spectroscopy}

We obtained optical spectroscopy on 2018 January 7, two days before radioactive maximum (14 d after discovery) using the Gemini MultiObject Spectrograph on the Gemini South telescope (GMOS-S; Hook et al. 2004). GMOS-S was configured using the R400 grating and a 1 arcsec long slit, with the detectors in $2 \times 2$ binning mode, covering a spectral range from 475 to $925 \mathrm{~nm}$ at a resolution of $\sim 900$. Data reduction was completed using the Gemini IRAF package. The data was first corrected for bias and flat-fielded using standard techniques. Cosmic rays were removed (van Dokkum 2001), as well as any detector bad columns. Note that the width of a cosmic-ray detection or bad column is small compared to the size of the resolved features in our spectra. The sky background was removed by first subtracting nodded pairs of images, followed by removing any residual by linear fitting the sky background near the target. One-dimensional spectra

${ }^{2}$ The lightcurves and spectra of SN 2016gkg and SN 1993J were gathered from https://sne.space/ (Guillochon et al. 2017). 


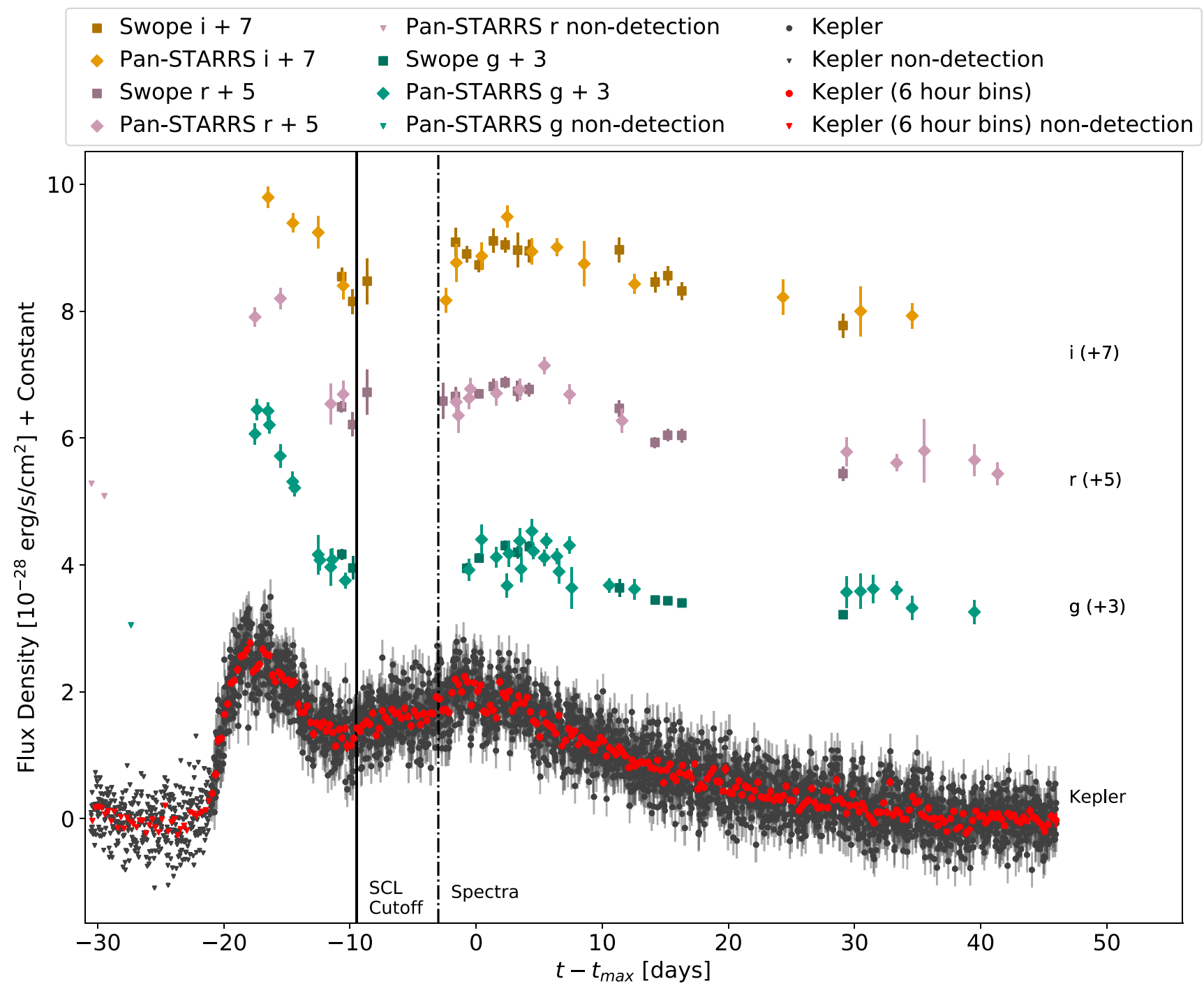

Figure 1. The combined Kepler/K2 and ground-based light curve of SN 2017jgh. The ground-based data is composed of Swope (Squares; Folatelli et al. 2010) and Pan-STARRS (Diamonds; Chambers et al. 2016) data. The black circles are the 30 min cadence Kepler/K2 data. In order to better see the Kepler/K2 light curve, we include $6 \mathrm{~h}$ bins of this light curve (red circles). The solid vertical line indicates where the SCL ends and the nuclear powered portion begins. The dashed vertical line shows when the spectrum of SN 2017jgh was taken.

were then produced using variance weighted extraction, utilizing a 1 arcsec spatial aperture. A similarly processed standard star, EG-131, was used to correct each extracted spectra for instrument response, prior to producing the final co-added spectrum. This Gemini baseline correction does not provide an absolute flux-calibrated spectrum, as the standard is not contemporaneous with the science observation, nor are slit-losses or second-order contamination a part of the standard correction. These calibration issues will likely compound at shorter wavelengths, which may be responsible for the increased flux around $5000 \AA$ in the SN 2017jgh spectrum compared to similar $\mathrm{SNe}$.

The reduced Gemini spectrum is shown in Fig. 4. The spectrum of SN 2017jgh is compared to both SN 1993J at $1 \mathrm{~d}$ before radioactive maximum (Jeffery et al. 1994; note that they state the spectra is "not photometrically accurate'), and SN $2016 \mathrm{gkg}$ at $2 \mathrm{~d}$ before radioactive maximum (Jha et al. 2016). The red (>6000 $)$ spectrum of SN 2017jgh is similar to both the SN 1993J spectrum, and the SN 2016gkg spectrum. Below $6000 \AA$ the spectrum of SN 2017jgh diverges from the other supernovae, likely due to the calibration issues we have described earlier.

We used the Supernova Identification (SNID; Blondin \& Tonry 2007) programme that classified SN 2017jgh as a Type IIb supernova. However, it is worth noting that with only one spectrum we do not have the robust IIb classification criteria of the observation of strong $\mathrm{He}$ features and the disappearance of $\mathrm{H}$ features.

\section{SHOCK COOLING CURVE MODELS}

Here, we summarize the formalism of the P15, P20, and SW17 analytical models.

\subsection{P15 Model}

The P15 model makes no assumptions about the density profile of the progenitor and assumes a simple expanding photosphere. P15 used these assumptions along with simple thermodynamic equations 

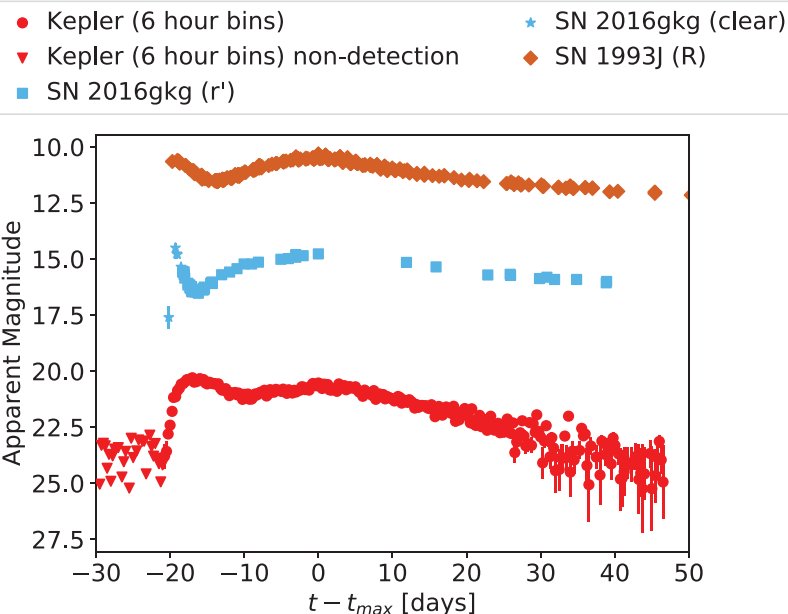

Figure 2. A comparison between the Kepler/K2 light curve of SN 2017jgh, the $r$ '-band photometry of SN 2016gkg (Arcavi et al. 2017), and the $R$-band photometry of SN 1993J (Richmond et al. 1994). Clear filter observations are also included for SN 2016gkg (Otero \& Buso 2016). The SCL does not have the sharp rise and decline of SN 2016gkg and appears similar to SN 1993J.

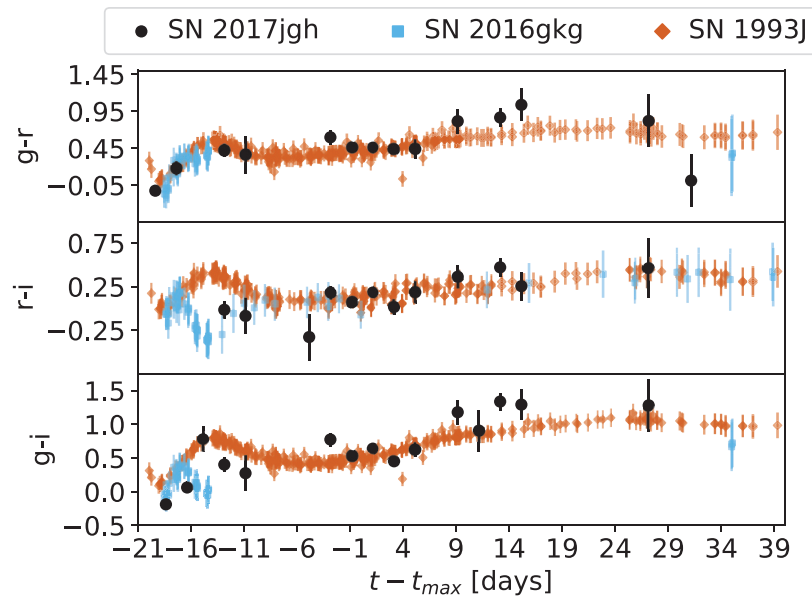

Figure 3. Colour evolution of SN 2017jgh (black), SN 2016gkg (blue), and SN 1993J (orange). The colour evolution of SN 2017jgh appears most similar with the SN 1993J evolution.

Table 1. Excerpt of light curve data for SN 2017jgh. The full light curve is available as supplementary material.

\begin{tabular}{lcccc}
\hline Time (MJD) & $\begin{array}{c}\text { Flux (erg } \\
\left.\mathrm{s}^{-1} \mathrm{~cm}^{-2}\right)\end{array}$ & $\begin{array}{c}\text { Flux Error (erg } \\
\left.\mathrm{s}^{-1} \mathrm{~cm}^{-2}\right)\end{array}$ & Band & Instrument \\
\hline 58117.33 & $1.50 \mathrm{e}-28$ & $9.16 \mathrm{e}-30$ & $r$ & Swope \\
58117.34 & $1.54 \mathrm{e}-28$ & $1.41 \mathrm{e}-29$ & $i$ & Swope \\
58117.35 & $1.17 \mathrm{e}-28$ & $8.38 \mathrm{e}-30$ & $g$ & Swope \\
58118.21 & $1.21 \mathrm{e}-28$ & $1.89 \mathrm{e}-29$ & $r$ & Swope \\
58118.22 & $1.15 \mathrm{e}-28$ & $1.95 \mathrm{e}-29$ & $i$ & Swope \\
58118.22 & $9.51 \mathrm{e}-29$ & $1.85 \mathrm{e}-29$ & $g$ & Swope \\
58119.36 & $1.72 \mathrm{e}-28$ & $3.54 \mathrm{e}-29$ & $r$ & Swope \\
58119.36 & $1.47 \mathrm{e}-28$ & $3.61 \mathrm{e}-29$ & $i$ & Swope \\
58125.37 & $1.58 \mathrm{e}-28$ & $2.82 \mathrm{e}-29$ & $r$ & Swope \\
58126.36 & $1.66 \mathrm{e}-28$ & $1.46 \mathrm{e}-29$ & $r$ & Swope \\
\hline
\end{tabular}

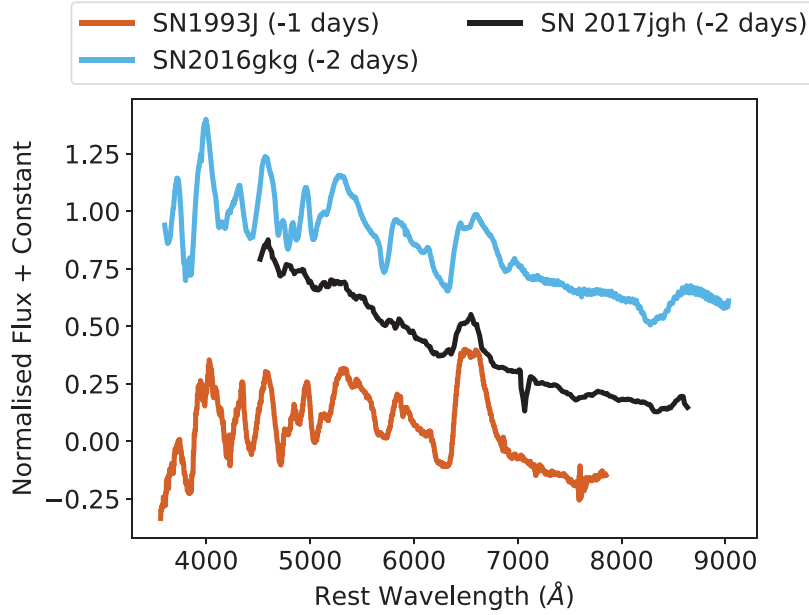

Figure 4. The spectrum of SN 2017jgh $2 \mathrm{~d}$ before radioactive maximum, compared to SN 1993J 1 d before radioactive maximum (Jeffery et al. 1994) and SN 2016gkg $2 \mathrm{~d}$ before radioactive maximum (Jha et al. 2016). All of the spectra have been normalized and then shifted in order to easily differentiate between them.

to derive the bolometric luminosity of an SCL. We follow Arcavi et al. (2017), who recast original equation for luminosity in P15 into the following equation that depends on the radius of the extended envelope $\left(R_{\mathrm{e}}\right)$, the mass of the envelope $\left(M_{e}\right)$, and the velocity of the envelope $\left(v_{\mathrm{e}}\right)$ :

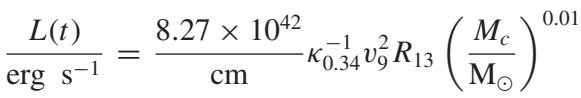

$$
\begin{aligned}
& \times \exp \left[\frac{-4.135 \times 10^{-11}}{\mathrm{~cm} \mathrm{~g} \mathrm{~g}^{-1} \mathrm{~s}^{-1}} t\left(t v_{9}+2 \times 10^{4} R_{13}\right)\right. \\
& \left.\times \kappa_{0.34}^{-1}\left(\frac{M_{c}}{\mathrm{M}_{\odot}}\right)^{0.01}\left(\frac{M_{e}}{0.01 \mathrm{M}_{\odot}}\right)^{-1}\right] \text {, }
\end{aligned}
$$

where $\kappa_{0.34}$ is the opacity in $0.34 \mathrm{~cm}^{2} \mathrm{~g}^{-1}, v_{9}$ is the envelope velocity in $10^{9} \mathrm{~cm} \mathrm{~s}^{-1}, R_{13}$ is the envelope radius in $10^{13} \mathrm{~cm}$, and $t$ is the time since explosion in seconds. Both $M_{e}$ and $M_{c}$ (the core mass) are in solar masses.

Assuming the emission is a blackbody with expanding radius $R(t)$ $=R_{\mathrm{e}}+v_{\mathrm{e}} t$, we can estimate the temperature as

$\frac{T(t)}{\mathrm{K}}=\left[\frac{L(t)}{4 \pi R^{2}(t) \sigma}\right]^{1 / 4}$,

where $\sigma$ is the Stefan-Boltzmann constant. With both the bolometric luminosity and the temperature defined, we are able to calculate the observed flux in arbitrary bands.

\subsection{P20 Model}

P20 provide an improvement upon the $\mathrm{P} 15$ model by considering a two-component model. This model consists of outer material with a steep velocity gradient, and inner material with a shallow velocity gradient. The transition between these layers occurs at the transition velocity $v_{t}$, related to the total energy of the extended material $E_{e}$ by

$v_{t}=\sqrt{\frac{(n-5)(5-\delta)}{(n-3)(3-\delta)}} \sqrt{\frac{2 E_{e}}{M_{e}}}$, 
where $t$ is the time in days, $n$ and $\delta$ are numerical factors that control the radial dependence of the outer and inner density structure, respectively. P20 show that the solution is not sensitive to the values of $n$ and $\delta$ as long as $n \gg 1$ and $\delta \gtrsim 1$. They suggest using typical value of $n \approx 10$ and $\delta \approx 1$. The P20 model has a two-component luminosity - the luminosity of the inner region and the luminosity of the outer region. Within the inner region, the luminosity is defined as

$$
\frac{L\left(t \leq t_{d}\right)}{\mathrm{erg} \mathrm{s}^{-1}} \approx 1.157 \times 10^{-5} \frac{E_{\mathrm{th}}\left(v_{t} t_{d}\right)}{t_{d}}\left(\frac{t_{d}}{t}\right)^{4 /(n-2)},
$$

where $t_{d}$ is the time when the SCL reaches the transition between the inner and outer regions and is equal to

$$
\frac{t_{d}}{\text { days }}=5.16 \times 10^{6} \sqrt{\frac{3 \kappa K M_{e}}{(n-1) v_{t} c}},
$$

where $c$ is the speed of light in $\mathrm{km} \mathrm{s}^{-1}$, and the numerical factor $K$ is equal to $\frac{(n-3)(3-\delta)}{4 \pi(n-\delta)}$; for our values of $n$ and $\delta, K=0.119 . E_{\mathrm{th}}\left(v_{t}, t_{d}\right)$ is the thermal energy at a given velocity and time:

$$
\frac{E_{\mathrm{th}}\left(v_{t}, t_{d}\right)}{\mathrm{erg}}=5.147 \times 10^{30} t_{d} \frac{\pi(n-1)}{3(n-1)} \frac{c R_{e} v_{t}^{2}}{\kappa} .
$$

The luminosity of the outer region is

$$
\frac{L\left(t \geq t_{d}\right)}{\mathrm{erg} \mathrm{s}^{-1}}=1.157 \times 10^{-5} \frac{E_{\mathrm{th}}\left(v_{t}, t_{d}\right)}{t_{d}} \exp \left[-\frac{1}{2}\left(\frac{t^{2}}{t_{d}^{2}}-1\right)\right] .
$$

In order to calculate the luminosity in any band we once again assume the SCL radiates as a blackbody, with a temperature:

$\frac{T}{\mathrm{~K}}=\left(\frac{L}{4 \pi r_{\mathrm{ph}}^{2} \sigma}\right)^{1 / 4}$.

Here, $r_{\mathrm{ph}}$ is the photospheric radius (the point where the optical depth $\tau$ is equal to $2 / 3$ ). The photospheric radius is also a two-component function dependent on $t_{\mathrm{ph}}$, the time when the photosphere reaches the transition between the inner and outer regions:

$$
\begin{aligned}
& \frac{t_{\mathrm{ph}}}{\text { days }}=5.16 \times 10^{6} \sqrt{\frac{3 \kappa K M_{e}}{2(n-1) v_{t}^{2}}}=\sqrt{\frac{c}{2 v_{t}}} t_{d}, \\
& \frac{r_{\mathrm{ph}}\left(t \leq t_{\mathrm{ph}}\right)}{\mathrm{cm}}=8.64 \times 10^{9}\left(\frac{t_{\mathrm{ph}}}{t}\right)^{2 /(n-1)} v_{t} t, \\
& \frac{r_{\mathrm{ph}}\left(t \geq t_{\mathrm{ph}}\right)}{\mathrm{cm}}=8.64 \times 10^{9}\left[\frac{\delta-1}{n-1}\left(\frac{t^{2}}{t_{\mathrm{ph}}^{2}}-1\right)+1\right]^{-1 /(\delta-1)} v_{t} t .
\end{aligned}
$$

The free parameters of the P20 model are $M_{e}$ in solar masses, $R_{e}$ in solar radii, and $v_{t}$ in $\mathrm{km} \mathrm{s}^{-1}$ (or $E_{e}$ as they are related by equation 4).

\subsection{SW17 Model}

Rabinak \& Waxman (2011) assume a polytropic progenitor density profile to derive an analytical form of the early light curve, characterized by the polytropic index $n$. This allows us to differentiate between progenitors with a convective envelope and a polytropic index of $n=3 / 2$, such as red supergiants (RSG), and progenitors with a radiative envelope and a polytropic index of $n=3$, such as blue supergiants (BSG). Sapir \& Waxman (2017) improve upon the Rabinak \& Waxman (2011) model by introducing an additional term that suppresses the luminosity at later times. This accounts for the shock phase days after the initial explosion, when the shock cooling emission begins to emerge from the inner layers. The SW17 bolometric luminosity (after the recasting done by Arcavi et al. $\left.(2017)^{3}\right)$ is

$$
\begin{aligned}
& \frac{L_{\mathrm{n}=3 / 2}(t)}{\mathrm{erg} \mathrm{s}^{-1}}=\frac{1.88 \times 10^{42}}{\mathrm{~cm}} \times \\
& \left(\frac{v_{s, 8.5} t^{2}}{f_{p} M \kappa_{0.34}}\right)^{-0.086} \frac{v_{s, 8.5}^{2} R_{13}}{\kappa_{0.34}} \times \\
& \exp \left(-\left\{\frac{1.67 t}{\frac{19.5}{\text { days cm }{ }^{-0.5} \mathrm{~s}^{-0.5}}\left(\kappa_{0.34} M_{e} v_{s, 8.5}^{-1}\right)^{0.5}}\right\}^{0.8}\right) \\
& \frac{L_{\mathrm{n}=3}(t)}{\operatorname{erg~s} \mathrm{s}^{-1}}=\frac{1.66 \times 10^{42}}{\mathrm{~cm}} \times \\
& \left(\frac{v_{s, 8.5} t^{2}}{f_{p} M \kappa_{0.34}}\right)^{-0.175} \frac{v_{s, 8.5}^{2} R_{13}}{\kappa_{0.34}} \times \\
& \exp \left(-\left\{\frac{4.57 t}{\frac{19.5}{\text { days cm } \mathrm{cm}^{-0.5} \mathrm{~s}^{-0.5}}\left(\kappa_{0.34} M_{e} v_{s, 8.5}^{-1}\right)^{0.5}}\right\}^{0.73}\right) .
\end{aligned}
$$

Here, $v_{s, 8.5}$ is the shock velocity in $10^{8.5} \mathrm{~cm} \mathrm{~s}^{-1}, M$ is equal to $M_{e}+$ $M_{c}$, and $t$ is the time since explosion in days. The factor $f_{p}$ is equal to $\sqrt{\frac{M_{e}}{M_{c}}}$ for $n=3 / 2$, and $0.08\left(M_{e} / M_{c}\right)$ for $n=3$.

The temperature is given as

$$
\begin{aligned}
& \frac{T_{n=3 / 2}(t)}{\mathrm{K}}=2.05 \times 10^{4} \times \\
& \left(\frac{v_{s, 8.5}^{2} t^{2}}{f_{p} M \kappa_{0.34}}\right)^{0.027}\left(\frac{R_{13}}{\kappa_{0.34}}\right)^{0.25} t^{-0.5} \\
& \frac{T_{n=3}(t)}{\mathrm{K}}=1.96 \times 10^{4} \\
& \times\left(\frac{v_{s, 8.5}^{2} t^{2}}{f_{p} M \kappa_{0.34}}\right)^{0.016}\left(\frac{R_{13}}{\kappa_{0.34}}\right)^{0.25} t^{-0.5} .
\end{aligned}
$$

Under the assumption that the emission is characterized by a blackbody, this once again allows us to calculate the luminosity in any band.

We make a number of assumptions for all models, which follow the assumptions made by Arcavi et al. (2017). We assume $\kappa_{0.34}=1$, typical for solar composition materials to exhibit electron scattering. We also set $M_{c}=1 M_{\odot}$. As stated in Arcavi et al. (2017), the early light curve is weakly dependent on this factor and our results are insensitive to it.

\section{FITTING THE SHOCK COOLING LIGHT CURVE}

For each model, we fit the gri bands and the binned Kepler/K2 data simultaneously. We fit from the first observation up to $-9.5 \mathrm{~d}$ before radioactive maximum. This was chosen to ensure our fits are not contaminated by emission from the main radioactive peak. For the $\mathrm{P} 15, \mathrm{SW} 17$, and P20 models, we fit the parameters $M_{e}, R_{e}, v_{e}$ or $v_{s}$ or $v_{t}$, respectively, and the offset time, $t_{\mathrm{off}}$, between the earliest observation and the start of the SCL.

We make use of the PYTHON EMCEE package (Foreman-Mackey et al. 2019), which provides an implementation of an affine-invariant ensemble Monte Carlo Markov Chain (MCMC) sampler. This algorithm attempts to produce an approximation of the posterior

${ }^{3}$ Note that Arcavi et al. (2017) had a typo in their equation 6 as the factor of 19.5 was included in the square root term within the exponent. This has been corrected here. 
Table 2. Uniform prior used for our MCMC fits.

\begin{tabular}{lccc}
\hline$R_{e}\left(\mathrm{R}_{\odot}\right)$ & $M_{e}\left(\mathrm{M}_{\odot}\right)$ & $v\left(\mathrm{~km} \mathrm{~s}^{-1}\right)$ & $t_{\text {off }}(\mathrm{d})$ \\
$0 \rightarrow 500$ & $0 \rightarrow 5$ & $0 \rightarrow 4 \times 10^{4}$ & $0 \rightarrow 15$ \\
\hline
\end{tabular}

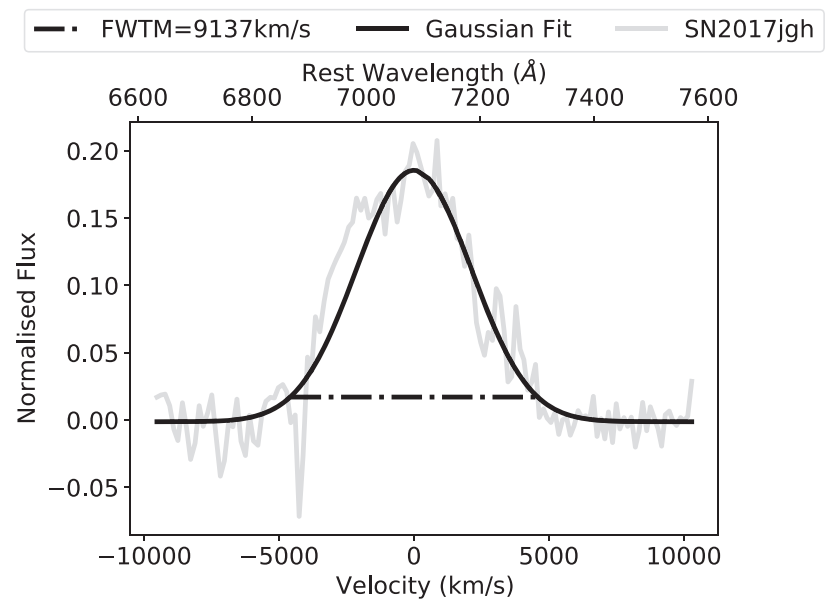

Figure 5. A Gaussian fit to the continuum subtracted $\mathrm{H} \alpha$ emission of SN 2017jgh. The solid black line is our Gaussian fit and the dashed line the full width at tenth maximum, used to estimate the velocity.

given a model, data, and a likelihood function which states how well the model fits the data. A number of initial positions are randomly chosen and evaluated with the likelihood function. From these initial positions, walkers traverse the parameter space, at each step deciding to either move to a random new position or stay at their current position based on how well the new parameter position fits the data. After each step, the walkers record their position in a chain. After a large number of steps, these chains will approximate the posterior. We use the reduced chi-squared as our likelihood function. We note that a chi-squared loss function naturally arises from a Gaussian log-likelihood, which assumed that each observations has white (uncorrelated) noise. The reduced chi-squared is inversely proportional to the degrees of freedom, which can be thought of as a regularization term to ensure that we are not biasing our fits to favour the higher cadence of the Kepler/K2 data.

For all fits, we use 500 walkers with a burn-in phase of 100 steps followed by 1000 additional steps. These were chosen after manually investigating the posterior and the walker chains to ensure they converged. The uniform priors used are provided in Table 2. These were initially chosen manually to fit the data while producing physically reasonable masses, radii, and velocities.

As an additional method of evaluating these fits, we compare their estimates of the velocity with that estimated from our spectrum. Following Jha et al. (2016), we approximate the expansion velocity of the supernova from the full width at tenth max of the $\mathrm{H} \alpha$ line. Fig. 5 shows a Gaussian fit to the $\mathrm{H} \alpha$ emission of SN 2017jgh in velocity space. The expansion velocity is measured to be $9100 \pm 470 \mathrm{~km} \mathrm{~s}^{-1}$.

The best fit to the light curve of SN 2017jgh is shown in Fig. 6 for each of our models of interest, the corner plots of each model are shown in Fig. 7, and the best-fitting values are given in Table 3. The best-fitting value is taken as the 50th percentile of the parameter posterior, with the 16th and 84th percentile as the lower and upper bounds, respectively. Note that this does not take into account the dependencies between parameters.
As can be seen in Fig. 6, P15 appears to perform the worst of the four models, with a small divergence at the start of the SCL rise and a larger deviation at about $-15 \mathrm{~d}$ before radioactive maximum. Additionally, the randomly drawn MCMC samples appear to diverge from the best fit. The offset time found by P15 $(-22.5 \mathrm{~d})$ is different to the offset time found by every other model (about $-21 \mathrm{~d}$ ) which accounts for the smaller deviation. The larger deviation and the divergence from the randomly drawn MCMC samples can be explained by the non-Gaussian posterior of P15's $R_{e}$ parameter. This non-Gaussian profile means that the median of the posterior does not lie at the maximum of the posterior. P20 suffers a similar issue of nonGaussianity, with the posterior of $M_{e}$ being multimodal (two peaks), although it does perform significantly better than P15 despite making similar assumptions. Unlike the SW17 models which have smaller residuals, neither the P15 nor P20 model make any assumption about the density of the progenitor which could account for their nonGaussian posteriors.

The SW17 $n=3 / 2$ and SW17 $n=3$ models have a reduced chisquared of 6.57 and 6.39 , respectively; these are betters fits to the ground-based data than P15 and P20 that have a reduced chi squared of 17.79 and 12.82, respectively. All models have relatively large reduced chi squared (with a value of 1 being desirable). This could be due to the inherent systematics in the Kepler/K2 data, or could be indicative of some physics not paramatrized by these models.

When comparing the best-fitting velocities to the velocity measured from the spectrum, $10200 \mathrm{~km} \mathrm{~s}^{-1}$, we see that the SW17 $n=$ $3 / 2$ once again best matches the data with a best fitting velocity of $\sim 8800 \mathrm{~km} \mathrm{~s}^{-1}$, as opposed to $\sim 14000 \mathrm{~km} \mathrm{~s}^{-1}, \sim 18000 \mathrm{~km} \mathrm{~s}^{-1}$, and $\sim 8100 \mathrm{~km} \mathrm{~s}^{-1}$ for P15, P20, and SW17 $n=3$, respectively. As such we conclude that SW17 $n=3 / 2$ is the best model for SN 2017jgh.

Overall it seems that the SW17 models are more physically accurate than P15 and P20 owing to their density assumption with SW17 $n=3 / 2$ being the best-fitting model for this supernova. This suggests that the progenitor of SN 2017jgh had an envelope radius of $\sim 130 R_{\odot}$ and an envelope mass of $\sim 0.50 \mathrm{M}_{\odot}$. This radius is similar to the progenitor radius of SN 1993J (Maund et al. 2004), and another well studied IIb supernova SN 2011dh $\left(\sim 200_{\odot}\right.$ Bersten et al. 2012), both of which are believed to have yellow supergiant progenitors. We conclude that the progenitor of SN 2017jgh was likely a yellow supergiant. This is reinforced by the similarities SN 2017jgh had with SN 1993J, in both its light curve and colour evolution.

\section{THE IMPORTANCE OF THE RISE}

As this is the first complete example of a high cadence shock cooling light curve, it provides an opportunity to evaluate how important the information contained in the rise is to getting an accurate fit. We perform fits to the ground-based lightcurve of SN 2017jgh without any Kepler/K2 data. The ground-based light curve contains no rise information and is quite similar in coverage and cadence to the light curves of SN 2016gkg and SN 1993J. Fig. 8 shows the results of these fits for the SW17 $n=3 / 2$ model, the model which best fit the full light curve. The best-fitting values are given in Table 4 , and a comparison between the posteriors of the ground-based fits and the fits to the full light curve are shown in Fig. 9.

Although the fits to the ground-based portion of the light curve appear reasonable, the extrapolated fits to the rise portion of the SCL are very poorly constrained. Additionally, the best fit does not match the Kepler/K2 light curve. Overall, this suggests that the decline of the SCL is not enough to constrain the rise of the SCL.

The best-fitting radius and mass of the ground-based fits $(\sim$ $150 \mathrm{R}_{\odot}$ and $\left.\sim 0.55 \mathrm{M}_{\odot}\right)$ are consistent with the radius and mass 


\begin{tabular}{lllll}
\hline i fits & $\square$ & Swope $\mathrm{i}+12$ & & Swope $\mathrm{g}+4$ \\
\hline Best fit & $\diamond$ & Pan-STARRS $\mathrm{i}+12$ & $\diamond$ & Pan-STARRS $\mathrm{g}+4$ \\
\hline $\mathrm{r}$ fits & $\square$ & Swope $\mathrm{r}+8$ & $\nabla$ & Pan-STARRS g non-detection \\
\hline g fits & $\diamond$ & Pan-STARRS $\mathrm{r}+8$ & $\diamond$ & Kepler $(6$ hour bins) \\
\hline Kepler (6 hour bins) fits & $\nabla$ & Pan-STARRS $r$ non-detection & $\nabla$ & Kepler (6 hour bins) non-detection
\end{tabular}
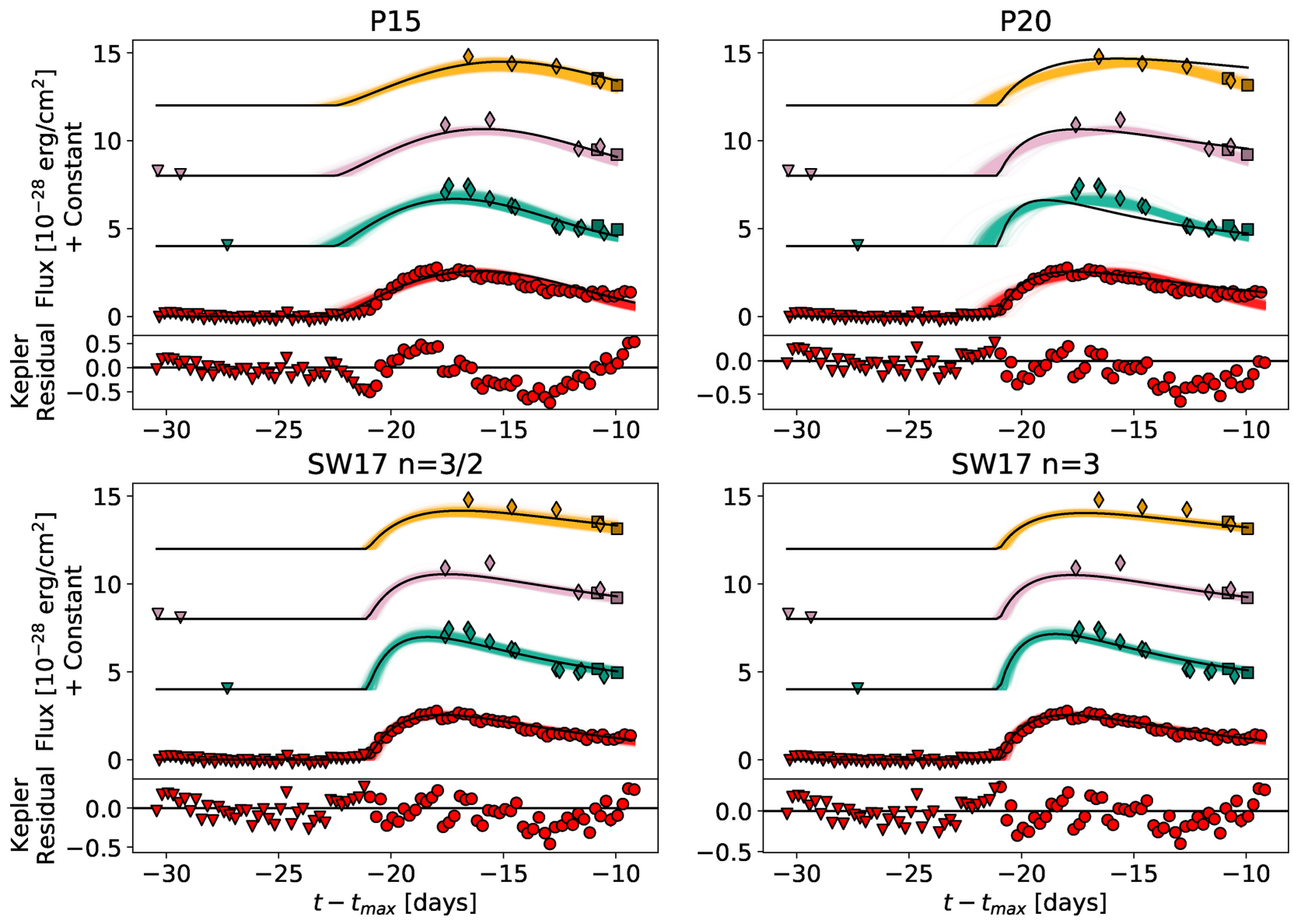

Figure 6. Model fits to the light curve of SN 2017jgh. The coloured lines are 1000 randomly drawn samples from the MCMC chain which give a visual understanding of the shape of the posterior. The black line is the median value of the posterior. Note that the median is taken from each parameter posterior independently so will differ from the randomly drawn samples. Additionally, the P15 and P20 models contain non-Gaussian posteriors so the median model is not an accurate reflection of the best-fitting model.

of the full light-curve fits $(\sim 130$ and $\sim 0.56)$; however, both the velocity and offset time are significantly different at $\sim 11000 \mathrm{~km}$ $\mathrm{s}^{-1}$ and $\sim-23 \mathrm{~d}$ compared to the full light curve fits of $\sim 8800 \mathrm{~km}$ $\mathrm{s}^{-1}$ and $\sim-21 \mathrm{~d}$. This suggests that the rise is required to constrain the velocity and explosion time; however, the envelope radius and envelope mass can be constrained with just the decline.

In order to estimate the numerical impact of the SCL rise on the quality of the fits, we calculate the percentage difference between the fits with and without the rise. We make use of bootstrap resampling to approximate the population mean and uncertainty. For both the full light-curve fit and the decline fit, we resample 10000 chains, each of which contain 500000 samples of the posterior. For each of these resampled chains, we find the median of the parameters, and then calculate the mean and standard deviation of these medians. The mean and percentage difference between the full light-curve fit and the decline fit is show in Table 5. The envelope mass is the least affected, with only a 1 per cent difference between the fits. The envelope radius, offset time, and velocity are more greatly affected, at 15 per cent, 20 per cent, and 25 per cent, respectively. These values provide a rough approximation for the systematic uncertainty of not having the rise of the SCL.

\section{CONCLUSIONS}

The high cadence Kepler/K2 light curve of SN 2017jgh provides a unique opportunity to investigate the complete shock cooling light curve of a Type IIb SNe. We fit the light curve with four models, the linearly expanding P15 model, the two-component P20 model, and the polytropic SW17 models with $n=3 / 2$ and $n=3$, modelling a red supergiant, and blue supergiant, respectively. 

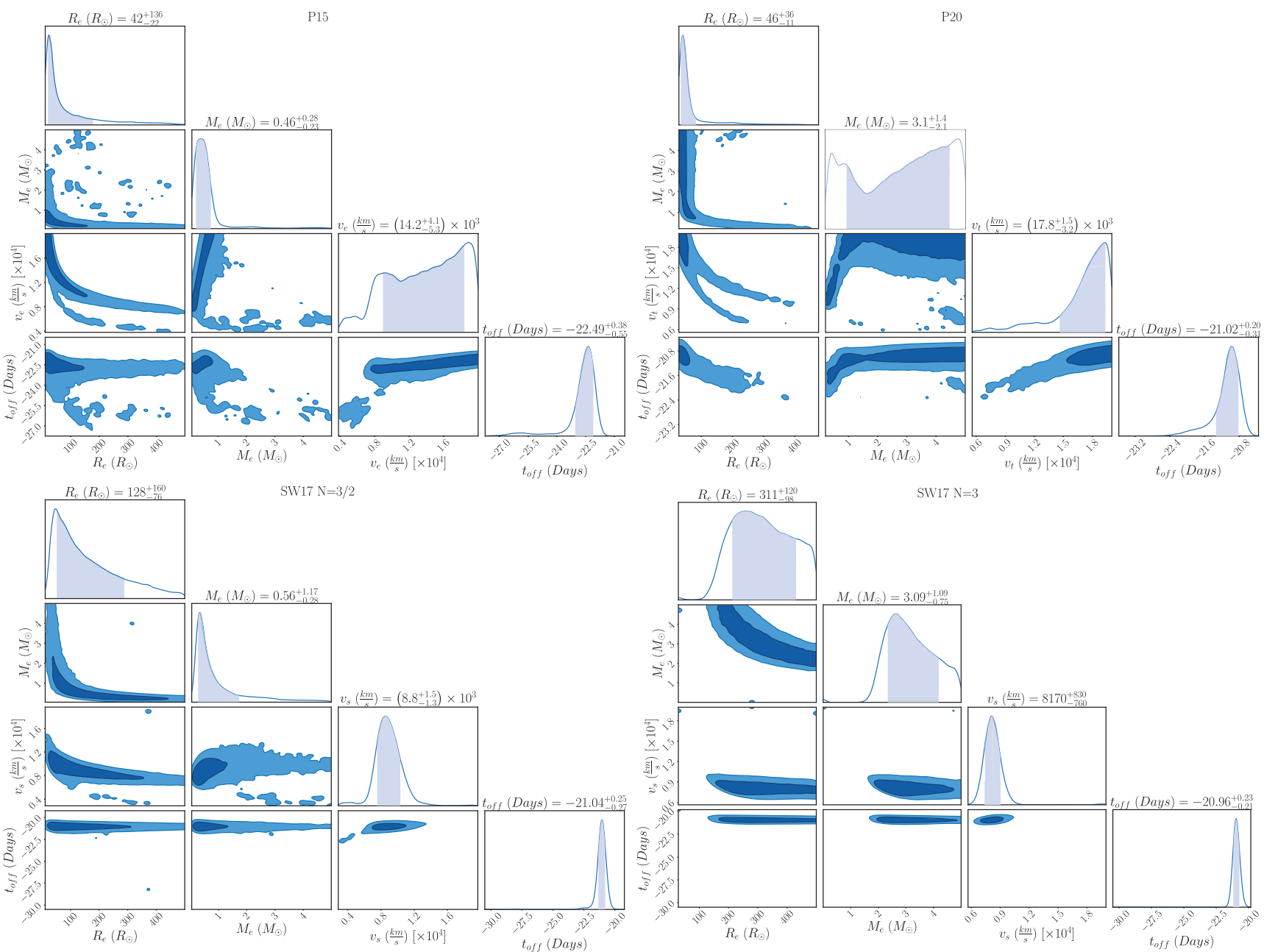

Figure 7. Corner plot \& results of our fits to the light curve of SN 2017jgh. The dark blue regions show the 1 sigma posterior. Created using the chainconsumer API. The best-fitting value was calculated using the cumulative method of chainconsumer, which takes the 50th percentile of the parameter posterior as the best fitting value. The 16th and 84th percentiles are then the lower and upper bounds, respectively. The shaded region of each posterior, and the joint posteriors, shows the lower and upper bounds (the 16th and 84th percentile). Note that $t_{\text {off }}$ is given in terms of days relative to radioactive maximum.

Table 3. Best-fitting parameters for each model, found by taking the 16th, 50th, and 84th percentile of each parameter as the lower bound, best value, and upper bound, respectively. Note that this does not take into account the dependencies between parameters. The reduced chi squared of the best-fitting value is also included. The SW17 models fit the lightcurve best, and have very similar velocity and offset times, however differ greatly in both the envelope radius and mass parameters. Since SW17 $n=3 / 2$ has a best-fitting velocity which is less than one standard deviation of the spectral velocity $\left(9100 \pm 470 \mathrm{~km} \mathrm{~s}^{-1}\right)$, we choose this as the preferred model for SN 2017jgh.

\begin{tabular}{lcccccc}
\hline Model & $R_{e}\left(\mathrm{R}_{\odot}\right)$ & $M_{e}\left(\mathrm{M}_{\odot}\right)$ & $v\left(10^{3} \mathrm{kms}^{-1}\right)$ & $t_{\text {off }}\left(t-t_{\max }\right)$ & Reduced $\chi^{2}$ & DOF \\
\hline P15 & $42.41_{-22.32}^{+135.66}$ & $0.46_{-0.23}^{+0.28}$ & $14.25_{-4.26}^{+4.14}$ & $-22.50_{-0.55}^{+0.38}$ & 17.79 & 4 \\
P20 & $45.82_{-11.04}^{+36.48}$ & $3.07_{-2.12}^{+1.37}$ & $17.79_{-3.16}^{+1.53}$ & $-21.02_{-0.31}^{+0.20}$ & 12.82 & 4 \\
SW17 $n=3 / 2$ & $127.82_{-76.11}^{+160.35}$ & $0.56_{-0.56}^{+1.17}$ & $8.81_{-1.26}^{+1.50}$ & $-21.05_{-0.27}^{+0.25}$ & 6.57 & 4 \\
SW17 $n=3$ & $310.62_{-97.59}^{+119.80}$ & $3.09_{-0.75}^{+1.09}$ & $8.17_{-0.76}^{+0.83}$ & $-20.96_{-0.21}^{+0.23}$ & 6.39 & 4 \\
\hline
\end{tabular}

After fitting the P15, P20, SW17 $n=3 / 2$, and SW17 $n=3$ models to SN 2017jgh, we found that the SW17 $n=3 / 2$ model provides the best fit (with a reduced $\chi^{2}$ of 6.57). This fit suggests that the progenitor of SN 2017jgh was a yellow supergiant with an envelope radius of $\sim 50-290 \mathrm{R}_{\odot}$, an envelope mass of $\sim 0-1.7 \mathrm{M}_{\odot}$, a shock velocity of $(7.5-10.3) \times 10^{3} \mathrm{~km} \mathrm{~s}^{-1}$, and an offset time of $\sim-21 \mathrm{~d}$ until radioactive maximum. SW17 $n=3 / 2$ is also best able to reproduce the measured shock velocity of $9100 \pm 470 \mathrm{~km} \mathrm{~s}^{-1}$.
Furthermore, we showed that the P15 and P20 models had difficulty reproducing the decline of the SCL, overestimating and underestimating it, respectively. This is possibly due to the lack of density information in these models. By comparison, both the SW17 $n=3$ and SW17 $n=3 / 2$ models follow the shape of the light curve better with very little deviation in the residual. The polytropic density model appears to be a better approximation for the true density of the shock cooling light curve. 


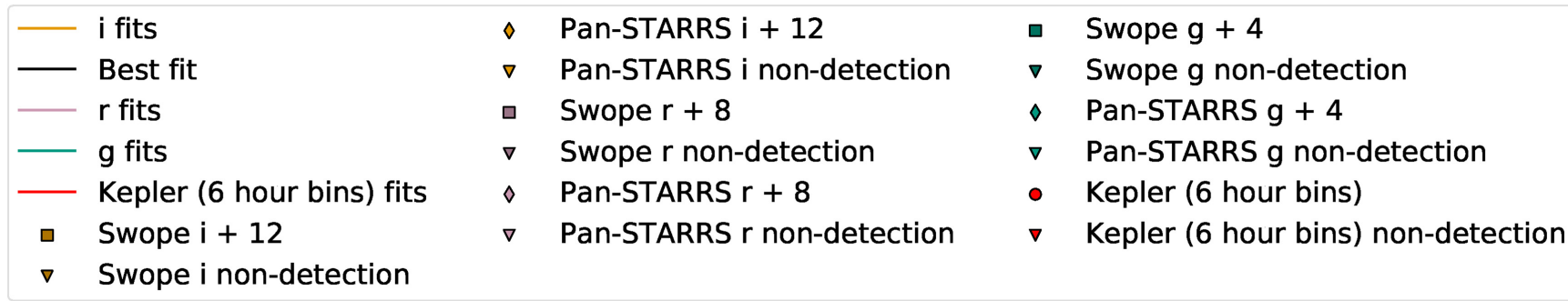

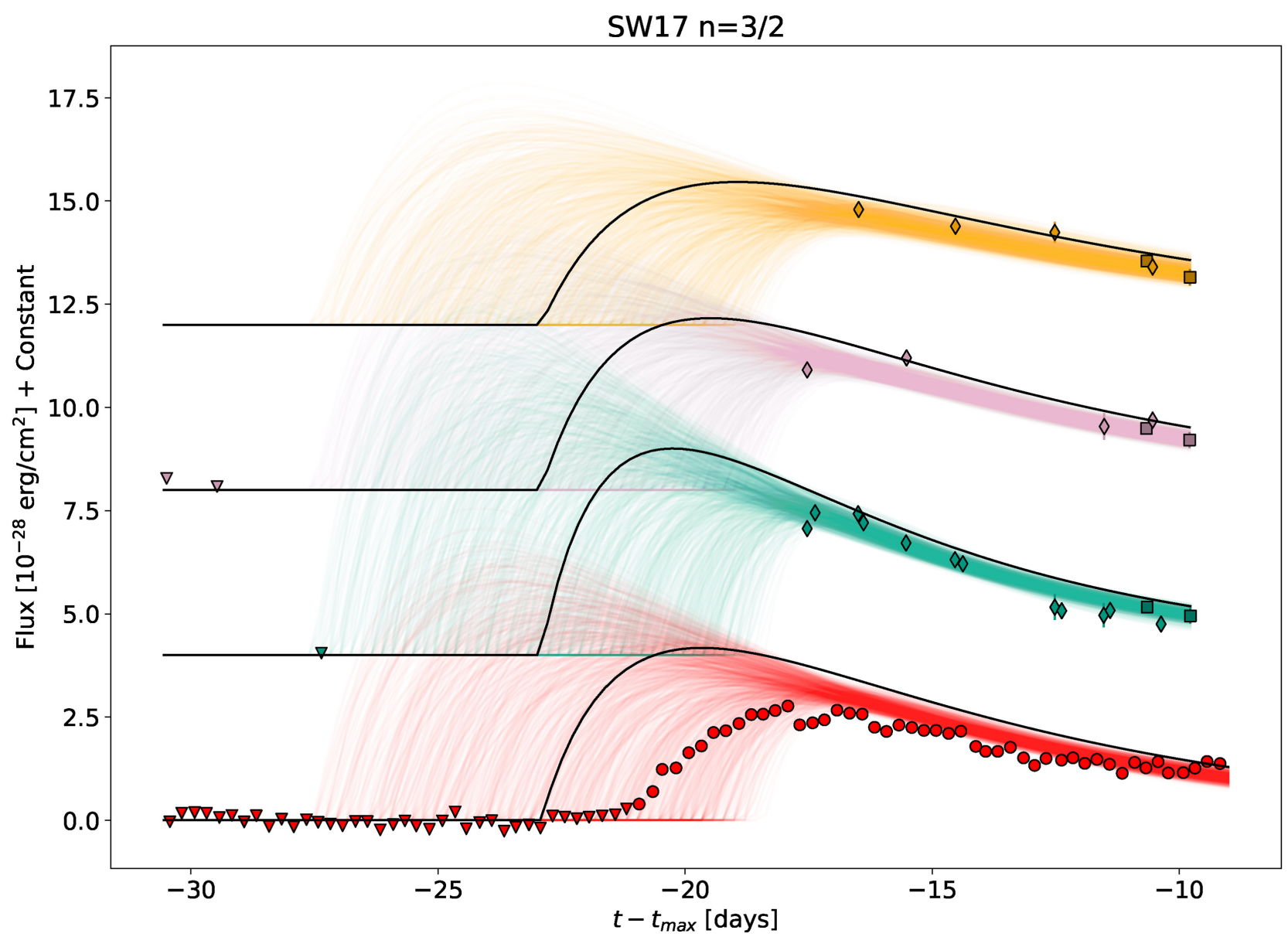

Figure 8. Fits to the ground-based light curve, ignoring all Kepler/K2 data. This emulates the conditions of most previous SCL observations. The coloured lines are 1000 randomly drawn samples from the MCMC chain which give a visual for the shape of the posterior. The black line is the median value of the posterior. Note that the median is taken from each parameter posterior independently so will differ from the randomly drawn samples. It is obvious that these fits do not constrain the rise time, and even though they seem reasonably when compared to the ground-based data, when applied to the Kepler/K2 data we see that they are quite inaccurate.

Table 4. Best-fitting parameters for fits to the ground-based light curve with the SW $17 n=3 / 2$ model, found by taking the 16th, 50th, and 84th percentile as the lower bound, best value, and upper bound, respectively. The reduced chi squared of the best-fitting value is also included.

\begin{tabular}{lllccc}
\hline Model & $R_{e}\left(\mathrm{R}_{\odot}\right)$ & $M_{e}\left(\mathrm{M}_{\odot}\right)$ & $v\left(\mathrm{~km} \mathrm{~s}^{-1}\right)$ & $t_{\text {off }}\left(t-t_{\max }\right)(\mathrm{d})$ & $\chi^{2}$ \\
SW17 $n=3 / 2$ & $150_{-100}^{+180}$ & $0.55_{-0.23}^{+1.16}$ & $\left(11.5_{-2.7}^{+3.7}\right) \times 10^{3}$ & $-22.9_{-3.1}^{+3.0}$ & 8.66 \\
\hline
\end{tabular}

In addition to determining the parameters of the progenitor of SN $2017 \mathrm{jgh}$, we use the complete SCL as an opportunity to investigate how important the rise of the shock cooling light curve is to the quality of fits. To do so, we fit the ground-based light curve of SN 2017 jgh ignoring any Kepler/K2 data. These fits were unable to constrain the explosion time and could not recover the Kepler/K2 lightcurve. This lead to a systematic offset in $v_{s}$, but both $R_{e}$ and $M_{e}$ seem mostly unaffected. In all models velocity and explosion time are degenerate, and radius and mass are degenerate. It appears that the rise constrains the offset time and velocity whilst the decline constrains the radius and mass. The rise is more sensitive to temperature and density (Piro 2015; Sapir \& Waxman 2017; Piro et al. 

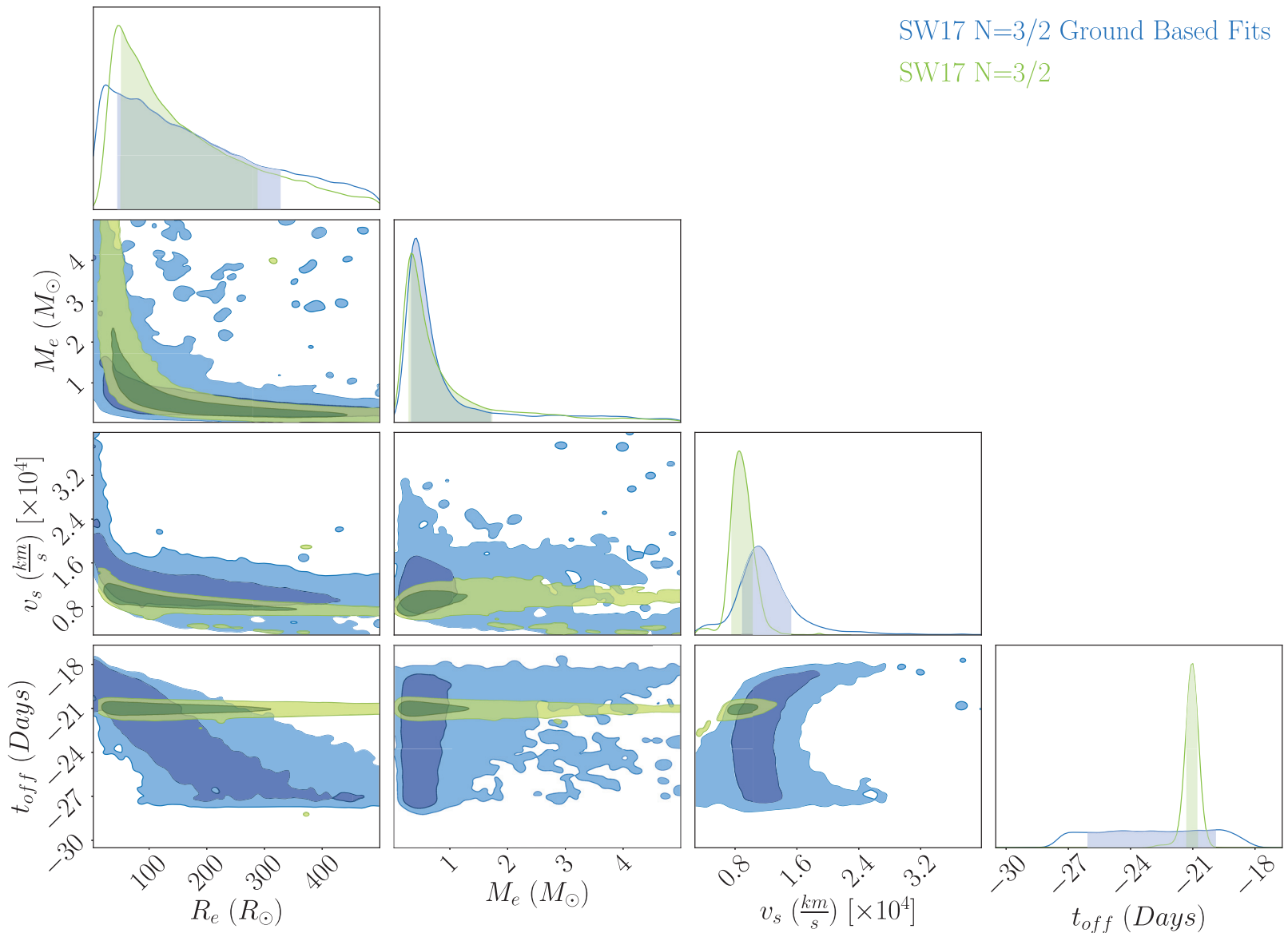

Figure 9. A comparison of the posteriors of the ground-based fits (blue) and the fits to the full lightcurve (green) for the SW17 $\mathrm{n}=3 / 2 \mathrm{model}$. The ground-based fits do not constrain the rise time and as such have a systematic offset in the $v_{s}$ parameter. However the ground-based fits do appear to constrain the $R_{e}$ and $M_{e}$ parameters at least as well as the full light-curve fits.

Table 5. Average parameter median over 10000 bootstrap resamples for both the full light-curve fit and decline fit, as well as the percentage difference between them.

\begin{tabular}{lcccc}
\hline & $R_{e}\left(\mathrm{R}_{\odot}\right)$ & $M_{e}\left(\mathrm{M}_{\odot}\right)$ & $v\left(10^{3}\right) \mathrm{km} \mathrm{s}^{-1}$ & $t_{\text {off }}\left(t-t_{\max }\right)(\mathrm{d})$ \\
\hline Full light-curve fit & $127.82 \pm 0.21$ & $0.56 \pm 0.0008$ & $8.81 \pm 0.0023$ & $9.5 \pm 0.00024$ \\
Decline fit & $149.11 \pm 0.25$ & $0.55 \pm 0.00055$ & $11.52 \pm 0.0047$ & $7.62 \pm 0.0062$ \\
Percentage difference & $15.38 \pm 0.67$ per cent & $1.13 \pm 0.49$ per cent & $26.67 \pm 0.14$ per cent & $22 \pm 0.23$ per cent \\
\hline
\end{tabular}

2020), so the shock velocity will also be sensitive to the shape of the rise.

Overall this suggests that the cadence and rise information contained within the Kepler/K2 lightcurve is invaluable to getting a good fit, specifically to constraining the offset time and shock velocity. This is less important for constraining the envelope radius and mass. Long term, high cadence surveys akin to Kepler/K2 will be essential for improving these analytical SCL models.

\section{SOFT WA RE}

NumPy (Harris et al. 2020), MatPlotLib (Hunter 2007), SciPy (Virtanen et al. 2020), AstroPy (The Astropy Collaboration et al. 2018), EMCEE (Foreman-Mackey et al. 2019), scikit-learn (Pedregosa et al. 2011), chainconsumer (Hinton 2016)

\section{FACIL I T IES}

Kepler (Howell et al. 2014), Gemini (Hook et al. 2004), Swope (Folatelli et al. 2010), PS1 (Chambers et al. 2016).

\section{ACKNOWLEDGEMENTS}

PA and BET acknowledge parts of this research was carried out on the traditional lands of the Ngunnawal people. We pay our respects to their elders past, present, and emerging. This paper includes data collected by the K2 mission. Funding for the K2 mission is provided by the NASA Science Mission directorate. KEGS is supported in part by NASA K2 cycle 4, 5, and 6 grants NNX17AI64G and 80NSSC18K0302, and 80NSSC19K0112, respectively. Pan-STARRS (PS1) is supported in part by the National 
Aeronautics and Space Administration under grants NNX12AT65G and NNX14AM74G. The PanSTARRS1 Surveys (PS1) and the PS1 public science archive have been made possible through contributions by the Institute for Astronomy, the University of Hawaii, the PanSTARRS Project Office, the Max-Planck Society and its participating institutes, the Max Planck Institute for Astronomy, Heidelberg and the Max Planck Institute for Extraterrestrial Physics, Garching, The Johns Hopkins University, Durham University, the University of Edinburgh, the Queen's University Belfast, the Harvard-Smithsonian Center for Astrophysics, the Las Cumbres Observatory Global Telescope Network Incorporated, the National Central University of Taiwan, the Space Telescope Science Institute, the National Aeronautics and Space Administration under grant NNX08AR22G issued through the Planetary Science Division of the NASA Science Mission Directorate, the National Science Foundation grant AST1238877, the University of Maryland, Eotvos Lorand University (ELTE), the Los Alamos National Laboratory, and the Gordon and Betty Moore Foundation Based on observations at Cerro Tololo InterAmerican Observatory, National Optical Astronomy Observatory (NOAO 2017B-0279; PI: A Rest, NOAO 2017B-0285; PI: A Rest), which is operated by the Association of Universities for Research in Astronomy (AURA) under a cooperative agreement with the National Science Foundation Based on observations obtained at the international Gemini Observatory, which is managed by the Association of Universities for Research in Astronomy (AURA) under a cooperative agreement with the National Science Foundation on behalf of the Gemini Observatory partnership: the National Science Foundation (United States), National Research Council (Canada), Agencia Nacional de Investigación y Desarrollo (Chile), Ministerio de Ciencia, Tecnología e Innovación (Argentina), Ministério da Ciência, Tecnologia, Inovações e Comunicações (Brazil), and Korea Astronomy and Space Science Institute (Republic of Korea). Observations in this program were obtained through program ID GS-2017B-LP-13. PA was supported by an Australian Government Research Training Program (RTP) Scholarship. BET and his group were supported by the Australian Research Council Centre of Excellence for All Sky Astrophysics in 3 Dimensions (ASTRO 3D), through project number CE170100013. The UCSC transient team is supported in part by NASA/K2 grants 80 NSSC18K0303 and 80NSSC19K0113, the Gordon \& Betty Moore Foundation, the Heising-Simons Foundation, and by a fellowship from the David and Lucile Packard Foundation to RJF. DOJ acknowledges support provided by NASA Hubble Fellowship grant HST-HF2-51462.001, which is awarded by the Space Telescope Science Institute, operated by the Association of Universities for Research in Astronomy, Inc., for NASA, under contract NAS5-26555. IA is a CIFAR Azrieli Global Scholar in the Gravity and the Extreme Universe Program and acknowledges support from that program, from the European Research Council (ERC) under the European Union's Horizon 2020 research and innovation programme (grant agreement number 852097), from the Israel Science Foundation (grant number 2752/19), from the United States - Israel Binational Science Foundation (BSF), and from the Israeli Council for Higher Education Alon Fellowship. MRD acknowledges support from the NSERC through grant RGPIN2019-06186, the Canada Research Chairs Program, the Canadian Institute for Advanced Research (CIFAR), and the Dunlap Institute at the University of Toronto. DAC acknowledges support from the National Science Foundation Graduate Research Fellowship under Grant DGE1339067. This project has been supported by the LP2018-7 Lendület grant of the Hungarian Academy of Sciences. LG acknowledges financial support from the Spanish Ministry of Science, Innovation and Universities (MICIU) under the 2019
Ramón y Cajal program RYC2019-027683 and from the Spanish MICIU project PID2020-115253GA-I00. BJS is supported by NASA grant 80NSSC19K1717 and NSF grants AST-1920392 and AST1911074. Support for TWSH was provided by NASA through the NASA Hubble Fellowship grant HST-HF2-51458.001-A awarded by the Space Telescope Science Institute, which is operated by the Association of Universities for Research in Astronomy, Inc., for NASA, under contract NAS5-26555. Research by SV is supported by NSF grants AST-1813176 and AST-2008108. LK acknowledges the financial support of the Hungarian National Research, Development and Innovation Office grant NKFIH PD-134784. LK and ZsB are supported by the Jànos Bolyai Research Scholarship of the Hungarian Academy of Sciences. The Konkoly team has been supported by the project 'Transient Astrophysical Objects' GINOP 2.3.2-152016-00033 of the National Research, Development and Innovation Office (NKFIH), Hungary, funded by the European Union. SWJ acknowledges support from US National Science Foundation award AST-1615455. This research has made use of the SVO Filter Profile Service (http://svo2.cab.inta-csic.es/theory/fps/) supported from the Spanish MINECO through grant AYA2017-84089. The LCO team is supported by NASA grant 80NSSC19K0119 and NSF grants AST1911225 and AST-1911151.

\section{DATA AVAILABILITY}

The data underlying this article are available in the article and in its online supplementary material.

\section{REFERENCES}

Abazajian K. N. et al., 2009, ApJS, 182, 543

Arcavi I. et al., 2011, ApJ, 742, L18

Arcavi I. et al., 2017, ApJ, 837, L2

Arcavi I., 2017, Hydrogen-Rich Core-Collapse Supernovae. Springer International Publishing, New York City, p. 239

Becker A., 2015, HOTPANTS: High Order Transform of PSF ANd Template Subtraction. Astrophysics Source Code Library (ascl:1504.004)

Bersten M. C. et al., 2012, ApJ, 757, 31

Bersten M. C. et al., 2018, Nature, 554, 497

Blondin S., Tonry J. L., 2007, in di Salvo T., Israel G. L., Piersant L., Burderi L., Matt G., Tornambe A., Menna M. T., eds, AIP Conf. Ser. Vol. 924, The Multicolored Landscape of Compact Objects and Their Explosive Origins. Am. Inst. Phys., New York, p. 312

Bryson S. T. et al., 2010, ApJ, 713, L97

Bufano F. et al., 2014, MNRAS, 439, 1807

Chambers K. C. et al., 2017, Transient Name Server Discovery Rep., $2017-$ 1494, 1

Chambers K. C. et al., 2019, The Pan-STARRS1 Surveys, preprint (arXiv:1612.05560)

Chevalier R. A., 1993, in Cassinelli J. P., Churchwell E. B., eds, ASP Conf. Ser. Vol. 35, Massive Stars: Their Lives in the Interstellar Medium. Astron. Soc. Pac., San Francisco, p. 393

Coulter D. A. et al., 2017, Science, 358, 1556

Dotson J. L. et al., 2018, Res. Notes Am. Astron. Soc., 2, 178

Fausnaugh M. M. et al., 2021, ApJ, 908, 51

Filippenko A. V., 1997, ARA\&A, 35, 309

Flewelling H. A. et al., 2020, ApJS, 251, 7

Folatelli G. et al., 2010, AJ, 139, 120

Foreman-Mackey D. et al., 2019, J. Open Source Softw., 4, 1864

Gal-Yam A., 2017, Observational and Physical Classification of Supernovae. Springer International Publishing, New York City, p. 195

Guillochon J., Parrent J., Kelley L. Z., Margutti R., 2017, ApJ, 835, 64

Harris C. R. et al., 2020, Nature, 585, 357

Hinton S., 2016, J. Open Source Softw., 1, 45 
Hook I. M., Jørgensen I., Allington-Smith J. R., Davies R. L., Metcalfe N., Murowinski R. G., Crampton D., 2004, PASP, 116, 425

Howell S. B. et al., 2014, PASP, 126, 398

Hunter J. D., 2007, Comput. Sci. Eng., 9, 90

Janka H.-T., 2012, Ann. Rev. Nucl. Part. Sci., 62, 407

Jeffery D. J. et al., 1994, ApJ, 421, L27

Jha S. W., Van Wyk V., Vaisanen P., 2016, Astron. Tel., 9528, 1

Kilpatrick C. D. et al., 2017, MNRAS, 465, 4650

Kilpatrick C. D. et al., 2018, MNRAS, 473, 4805

Krisciunas K. et al., 2017, AJ, 154, 211

Kumar B. et al., 2013, MNRAS, 431, 308

Liu Y.-Q., Modjaz M., Bianco F. B., Graur O., 2016, ApJ, 827, 90

Magnier E. A. et al., 2020, ApJS, 251, 3

Magnier E. A., Liu M., Monet D. G., Chambers K. C., 2008, in Jin W. J., Platais I., Perryman M. A. C., eds, A Giant Step: from Milli- to Microarcsecond Astrometry, Vol. 248. Cambridge University Press, Cambridge, United Kingdom, p. 553

Maund J. R., Smartt S. J., Kudritzki R. P., Podsiadlowski P., Gilmore G. F., 2004, Nature, 427, 129

Morales-Garoffolo A. et al., 2014, MNRAS, 445, 1647

Naiman B. V., Sabach E., Gilkis A., Soker N., 2019, MNRAS, 491, 2736

Nakar E., Piro A. L., 2014, ApJ, 788, 193

Nakar E., Sari R., 2010, ApJ, 725, 904

Nomoto K., Suzuki T., Shigeyama T., Kumagai S., Yamaoka H., Saio H., 1993, Nature, 364, 507

Otero S., Buso V., 2016, Trans. Name Ser. Discovery Rep., 2016, 1

Pedregosa F. et al., 2011, J. Mach. Learn. Res., 12, 2825

Piro A. L., 2015, ApJ, 808, L51 (P15)

Piro A. L., Haynie A., Yao Y., 2021, Shock Cooling Emission from Extended Material Revisited, Vol. 909, American Astronomical Society, Washington, DC, p. 209(P20)

Podsiadlowski P., 1992, PASP, 104, 717

Quintana E. V. et al., 2010, Software and Cyber Infrastructure for Astronomy. Proceedings of the SPIE, Bellingham WA, p. 77401X

Rabinak I., Waxman E., 2011, ApJ, 728, 63

Rest A. et al., 2005, ApJ, 634, 1103

Richmond M. W., Treffers R. R., Filippenko A. V., Paik Y., Leibundgut B., Schulman E., Cox C. V., 1994, AJ, 107, 1022

Ricker G. R. et al., 2014, J. Astron. Telesc. Instr. Syst., 1, 014003

Rodrigo C., Solano E., 2020, The SVO Filter Profile Service. Contributions to the XIV.0 Scientific Meeting (virtual) of the Spanish Astronomical Society, p. 182

Rodrigo C., Solano E., Bayo A., 2012, SVO Filter Profile Service Version 1.0, IVOA Working Draft 15 October 2012

Ryan S., ed., 2006, The Advanced Maui Optical and Space Surveillance Technologies Conference, AMOS Conference, Kihei USA

Sapir N., Waxman E., 2017, ApJ, 838, 130 (SW17)

Schlegel D. J., Finkbeiner D. P., Davis M., 1998, ApJ, 500, 525

Scolnic D. et al., 2015, ApJ, 815, 117

Shaya E. J., Olling R., Mushotzky R., 2015, AJ, 150, 188

Sravan N., Marchant P., Kalogera V., 2019, ApJ, 885, 130

Sravan N., Marchant P., Kalogera V., Milisavljevic D., Margutti R., 2020, ApJ, 903, 70

Stumpe M. C. et al., 2012, PASP, 124, 985

The Astropy Collaboration et al., 2018, AJ, 156, 123

Tonry J. L. et al., 2012, ApJ, 750, 99

Vallely P. J., Kochanek C. S., Stanek K. Z., Fausnaugh M., Shappee B. J., 2021, MNRAS, 500, 5639

Van Cleve J. E., Caldwell D. A., 2016, Kepler Instrument Handbook. Kepler Science Document KSCI-19033-002, Washington, DC

van Dokkum P. G., 2001, PASP, 113, 1420

Virtanen P. et al., 2020, Nature Methods, 17, 261

Waxman E., Katz B., 2017, Shock Breakout Theory. Springer, Cham, Salmon Tower, New York City, p. 967

Woosley S. E., Langer N., Weaver T. A., 1993, ApJ, 411, 823

Yoon S.-C., Dessart L., Clocchiatti A., 2017, ApJ, 840, 10

\section{SUPPORTING INFORMATION}

Supplementary data are available at MNRAS online.

\section{AT2017jgh.fits jghFinal.txt}

Please note: Oxford University Press is not responsible for the content or functionality of any supporting materials supplied by the authors. Any queries (other than missing material) should be directed to the corresponding author for the article.

${ }^{1}$ Mt Stromlo Observatory, The Research School of Astronomy and Astrophysics, Australian National University, ACT 2601, Australia

${ }^{2}$ National Centre for the Public Awareness of Science, Australian National University, Canberra, ACT 2611, Australia

${ }^{3}$ The ARC Centre of Excellence for All-Sky Astrophysics in 3 Dimensions (ASTRO 3D), Canberra, ACT 2611, Australia

${ }^{4}$ Space Telescope Science Institute, 3700 San Martin Dr., Baltimore, MD 21218, USA

${ }^{5}$ Department of Physics and Astronomy, The Johns Hopkins University, 3400 North Charles Street, Baltimore, MD 21218, USA

${ }^{6}$ The Observatories of the Carnegie Institution for Science, 813 Santa Barbara St., Pasadena, CA 91101, USA

${ }^{7}$ School of Mathematics and Physics, University of Queensland, Brisbane, QLD 4072, Australia

${ }^{8}$ Centre for Gravitational Astrophysics, College of Science, The Australian National University, ACT 2601, Australia

${ }^{9}$ Gemini Observatory/NSF's NOIRLab, Casilla 603, La Serena, Chile

${ }^{10}$ University of Illinois at Urbana-Champaign, 1003 W. Green Str, IL 61801, USA

${ }^{11}$ Centre for Astrophysical Surveys, National Centre for Supercomputing Applications, Urbana, IL 61801, USA

${ }^{12}$ Astronomy Department, University of Maryland, College Park, MD 20742, USA

${ }^{13}$ University of Notre Dame, Notre Dame, IN 46556, USA

${ }^{14}$ Department of Astronomy and Theoretical Astrophysics Center, University of California, Berkeley, CA 94720, USA

${ }^{15}$ Department of Physics, University of California, Berkeley, CA 94720, USA

${ }^{16}$ Lawrence Berkeley National Laboratory, Berkeley, CA 94720, USA

${ }^{17}$ Department of Astronomy, Columbia University, New York, NY 10027, USA

${ }^{18}$ Cerro Tololo Inter-American Observatory, NSF's National Optical-Infrared Astronomy Research Laboratory, Casilla 603, La Serena, Chile

${ }^{19}$ The School of Physics and Astronomy, Tel Aviv University, Tel Aviv 69978, Israel

${ }^{20}$ CIFAR Azrieli Global Scholars program, CIFAR, Toronto, Canada

${ }^{21}$ David A. Dunlap Department of Astronomy and Astrophysics, University of Toronto, 50 Str George Street, Toronto, Ontario, M5S 3H4, Canada

${ }^{22}$ Department of Astronomy and Astrophysics, University of California, Santa Cruz, CA 95064, USA

${ }^{23}$ Department of Astronomy, University of Texas at Austin, Austin, TX 78712, USA

${ }^{24}$ Centro de Astronomía (CITEVA), Universidad de Antofagasta, Avenida U. de Antofagasta 02800, Antofagasta, Chile

${ }^{25}$ Las Campanas Observatory, Carnegie Observatories, Casilla 601, La Serena, Chile

${ }^{26}$ Center for Interdisciplinary Exploration and Research in Astrophysics (CIERA), Evanston, IL 60201, USA

${ }^{27}$ Department of Physics and Astronomy, Northwestern University, Evanston, IL 60208, USA

${ }^{28}$ Max-Planck-Institut fur Astrophysik, Karl-Schwarzschild-Str 1, D-85748 Garching bei München, Germany

${ }^{29}$ Centre for Astrophysics Research, School of Physics, Astronomy and Mathematics, University of Hertfordshire, College Lane, Hatfield AL10 9AB, $U K$ 
${ }^{30}$ Institute for Astronomy, University of Hawaii at Manoa, 2680 Woodlawn Drive, Honolulu, HI 96822, USA

${ }^{31}$ Astrophysics Research Centre, School of Mathematics and Physics, Queen's University Belfast, BT7 1NN, UK

${ }^{32}$ Exoplanets and Stellar Astrophysics Laboratory, Code 667, NASA Goddard Space Flight Center, Greenbelt, MD 20771, USA

${ }^{33}$ University of Maryland, Baltimore County, 1000 Hilltop Circle, Baltimore, MD 21250, USA

${ }^{34}$ Bay Area Environmental Research Institute, P.O. Box 25, Moffett Field, CA 94035, USA

${ }^{35}$ NASA Ames Research Center, Moffett Field, CA 94035, USA

${ }^{36}$ School of Physics, The University of Melbourne, Parkville, VIC 3010, Australia

${ }^{37}$ Konkoly Observatory, Research Centre for Astronomy and Earth Sciences, Eötvös Lorànd Research Network (ELKH), Konkoly Thege Miklòs ùt 15-17, Budapest H-1121, Hungary

${ }^{38}$ MTA CSFK Lendület Near-Field Cosmology Research Group, Konkoly Thege Miklós út 15-17, Budapest H-1121, Hungary

${ }^{39}$ Coral Towers Observatory, Cairns, QLD 4870, Australia

${ }^{40}$ Department of Physics and Astronomy, Texas A\&M University, 4242 TAMU, College Station, TX 77843, USA

${ }^{41}$ Institute of Space Sciences (ICS, CSIC), Campus UAB, Carrer de Can Magrans, s/n, E-08193 Barcelona, Spain

${ }^{42}$ Department of Physics, University of California, Santa Barbara, CA 93106, USA

${ }^{43}$ Las Cumbres Observatory, 6740 Cortona Dr, Suite 102, Goleta, CA 93117 , USA
${ }^{44}$ Department of Physics and Astronomy, Rutgers the State University of New Jersey, 136 Frelinghuysen Road, Piscataway, NJ 08854, USA

${ }^{45}$ Institute of Physics, University of Szeged, Dom ter 9, Szeged, H-6720, Hungary

${ }^{46}$ Steward Observatory, University of Arizona, 933 North Cherry Avenue, Tucson, AZ 85721, USA

${ }^{47}$ Departamento de Astronomía y Astrofísica, Universidad de Valencia, E46100 Burjassot, Valencia, Spain

${ }^{48}$ Observatorio Astronómico, Universidad de Valencia, E-46980 Paterna, Valencia H-1518, Spain

${ }^{49}$ Graduate Institute of Astronomy, National Central University, 300 Jhongda Road, 32001 Jhongli, Taiwan

${ }^{50}$ Physics Department and Tsinghua Center for Astrophysics (THCA), Tsinghua University, Beijing 100084, China

${ }^{51}$ ELTE Eötvös Loránd University, Institute of Physics, Budapest, Hungary

${ }^{52}$ Department of Physics and Astronomy, University of California, 1 Shields Avenue, Davis, CA 95616, USA

${ }^{53}$ Beijing Planetarium, Beijing Academy of Science and Technology, Beijing 100044, China

${ }^{54}$ Physics Department, Tsinghua University, Beijing 100084, China

${ }^{55}$ European Southern Observatory, Karl-Schwarzschild-Strasse 2, D-85748

Garching bei München, Germany

This paper has been typeset from a $\mathrm{T}_{\mathrm{E}} \mathrm{X} / \mathrm{L} \mathrm{T} \mathrm{E} \mathrm{X}$ file prepared by the author. 